\title{
XXIII.
}

\section{Bericht über die Jahressitzung des Vereins der deutschen Irrenärzte am 16. und 1\%. September 1884 in Leipzig.}

Der Verein tagte in dem Auditorium der Irrenklinik.

Vorstand: von Gudden, Laehr, Nasse, Schüle, Westphal.

Vorsitzender: Nasse.

Tagesordnung.

No. 1. Bericht des Vorstandes, betreffend frühere Vereinsbeschlüsse.

Den Beschlüssen des Vorstandes vom 15. Februar 1882 über die Einführung neuer Zäblkarten ist das Königl. preussische Ministerium des Cultus und des Innern am 19. Juni im Wesentlichen beigetreten.

Die Eingabe an den Reichskanzler, betreffend die Aufnahmeder Psychiatrie in die Staatsprüfung ist vom Bundesrath durch Antwort vom 26. Juni 1883 abgelehnt worden.

Die Vorschläge des Vereins für die Behandlung geisteskranker Verbrecher sind noch nicht zum Abschluss gelangt. Der Verein sieht daher vorläufig von weiteren Schritten ab.

No. 2. Referat Sander's (Dalldorf) über die Frage, ob und unter wolchen Umständen Geistesstörung als Ehescheidungsgrund betrachtet werden soll.

Zwischen Geistesstörung und anderen Krankheiten besteht ein principieller Unterschied bohufs der Ehoscheidungsfrage nicht. Geisteskrankheiten vor Allem berechtigen aber zur Ehescheidung wogen ihres starken Procentsatzes an Unheilbarkeit, wegen ihrer langen Dauer, wegen ihres frühen Beginnens und endlich weil sie die geistige Gemeinschaft stören. Der Hauptgrund, welcher die Leute zur Ehescheidung bei Geistesstörung veranlasst, ist die Noth. die hüflose Lage, in welche der gesunde Theil bei Geisteskrankheit des anderen geräth, denn vorwiegend sind es Arme, welche auf Ehescheidung antragen. Bei günstigen äusseren Verhältnissen lassen sich die durch Geisteskrankheit gesetzten Nachtheilo noch ausgleichen. Statistische Erfahrungen beweisen uns die Seltenheit des Vorkommens der Ehescheidung bei geistes- 
kranken Gatten. Unter den an den Berliner Landgerichten angestrengten Ehescheidungsprocessen fallen nur 5-6 pCt. auf Geisteskrankheit; 2 waren gegen den Ehemann gerichtet, bis auf einen Fall waren alle Parteien arm.

Bei den Einwänden, welche gegen die Ehescheidung bei geisteskranken Gatten zu erheben wären, sind vornehmlich ärztliche Gründe in's Feld zu führen: Schwierigkeit der Prognose bei Geisleskrankheiten, Spätheilungen und Heilbarkeit der Paralyse. Verf. giebt zu, dass letztere Fälle vorkommen, hat aber selbst nie solche gesehen. Spätheilungen sind sehr fraglich. Beide Fragen thun aber dem Princip der Gesetzgebung keinen Eintrag. Vor Allem kommt es darauf an, die Unheilbarkeit der Kranken nach längerer Beobachtung in einer Anstalt zn bestimmen; drei Jahre dürften in den meisten Fällen genügen. Ferner ist die materielle Lage des Kranken in jedem Falle sicher zu stellen. Das Verfahren bei der Ehescheidung soll sich all das Entmündidungsverfahren anschliessen: auch hier ist die Zahl der zu ernennenden Sachverständigen dem Richter anbeimzugeben. Vortragender resumirt seinen Bericht dahin :

1. Principielle Zulässigkeit der Ehescheidung aus religiösen, ethischen Gründen ist nicht Aufgabe des Vereins.

2. Unheilbare Geistesstörung ist ein Ehescheidungsgrund.

3. Das Verfahren soll analog sein dem der Entmündigung.

4. Die Interessen des Kranken müssen gewahrt werden.

5. Ein Antrag auf Nichtigkeit der Ehe ist nur dann zu stellen, wenn ein Theil zur Zeit der Eheschliessung geisteskrank und dem anderen dieses nicht bekannt war. Letzter Punkt erfordert besondere Bestimmungen.

\section{Discussion:}

v. Gudden macht darauf aufmerksam, dass bei der weiteren Vorverhandlung der Vorstand angenommen hat. dass die Frage des Referenlen bereits zu weit gegangen sei. Der Verein beschränkt sich nur auf die Frage, wie bei der gesetzlichen Zulässigkeit der Ehescheidung bei unheilbaren Irren das technisch ärztliche Verfahren zu handhaben sei.

Sander hält nicht für recht, dass der Verein sich diese Beschränkung auferlegt, denn warum sollten die Irrenärzte nicht vor Allem diese Frage* entscheiden?

Nach kurzer Debalte beschliesst der Verein, über den Salz nicht weiter abzustimmen und die weitere Verhandlung des Gegenstandes fallen zu lassen.

No. 3. Sander-Dalldorf. Referat über die Frage. in wie weit Milglieder von Kranken-, Invaliden-und anderen Kassen beim Eintritt von Geistesstörung Nachtheile in der Geltendmachung ihrer Rechte erleiden.

Die bisherigen Verfügungen darüber sind unzulänglich und unzweckmässig, sowohl die Bestimmung der einzelnen Kassen, als auch die gesetzliche Vorschrift.

Der Verein der deutschen Irrenärzte erklärt unter Berücksichtigung des 
Umstandes, dass nach dem Gesetze; betreffend die Krankenversicherang, eine principielle Ausnahme zu Ungunsten der Geisteskranken nicht mehr statthaft ist, seinen vorjährigen Beschluss in dieser Beziehung für erledigt, ersucht aber seine Mitglieder, die Fälle, in denen Geisteskranke der ihnen zustehenden Rechte durch Verkennen der krankhaften Erscheinungen verlustig gehen, besonders in's Auge zu fassen und sie möglichst zu verhindern.

Der Beschluss wird angenommen.

No. 4. Laehr-Schweizerhof. Bericht äber die Fortschrittedes deutchen Irrenanstaltswesens in den letzten Jahren.

Verfasser giebt ein arschauliches Bild über Frequenz, bauliche Verbesserungen und Erweiterungen sämmtlicher Irrenanstalten des deatschen Reichs. Fülle und Eigenarligkeit des Materials gestalten nicht, den Vortrag in ein Referat zusammenzudrängen.

Der Vortrag bliob ohne Discussion.

No. 5. Flechsig-Leipzig. Zur gynäkologisehen Bohandlung hysterischer Personen. (Der Vortrag ist in extenso veröffentlicht im Neurologischen Centralblatt 1884 No. 19 und No. 20.)

Aus der Literatur sind 10 Fälle belsannt, in welchen die Castration gegeu Psychosen gemacht wurde: 4 mit günstigen Erfolge, 3 ohne Resultat, 3 mit Verschlimmerung.

F lechsig theilt aus eigener Beobachtung zwei Fälle mit.

1. Fall. Unverheirathetes, 32 Jahre alles Mädchen. Im 19. Jahre Elregungszustand, im 24. Krämpfe, im 28. Jahre Beginn der hysterischen Beschwerden : zunächst Depressionszustand wit Neigung zum Suicidium, Verfolgungsideen, hysterische Delirien. Krampfanfälle: Trismus, Singultus, Erbrechen, Retentio urinae. Halbseitige Anästliesie.

Maniakalische und Depressionszustände wechseln. Intelligenzdefect. Nach 2/, jährigem Bestehen dieses Zustandes kam Patientin in die Klinik.

Status: Somatisch, leichtes Lungenemphysem.

Im linken Parametrium alte Parametritis.

Vollkommene Hemiparese links, selbst Facialis.

Steigerung der Sehnenreflexe.

Ophthalmoskopisch: Staphylom auf einer Seite.

Krampfanfälle: wechselhaft in ihren Erscheinungen. Hochgradige Ovarie links. Bei der Menstruation Steigerung der Anfälle.

Psychisch wechselndos Verhalten. Vorwiegend Dämmerzustände.

Die Operation schien indicirt wegen der der alten Parametritis (narbige Stränge) und der dadurch hervorgerufenen Steigerung der Beschwerden während der Menses. Es wurde daher die Castration jm Juli 1883 ausgefübrt. Ovarien klein, im Parenchym derselben viele kleine Cysten. Beim Herausziehen der Ovarien hatte die Kranke trotz der tiefen Narcose Anfälle.

Heilung verlief gut; bereits nach 12 Tagen konnte die Kranke das Bett verlassen. Nach 14 Tagen traten jedoch bereits wieder hysterische Delirien auf. Die Anfälle hatten jetzt einen anderen Charakter, als vor der Operation. Bis zur 21. Woche nach der Operation Steigerung der psychischen Erschei- 
nungen. Erst im December nach einem heftigen Verwirrungszustande trat eine Besserung ein. Seit jener Zeit fühlt sich die Kranke vollkommen wohl.

Verfasser schreibt diesen günstigen Erfolg einzig und allein der Operation zu; das Recidiv wäre nicht als Gegenbeweis anzusehen. Für den guten Effect unmittelbar nach der Operation wäre vielleicht die protrahirte tiefe Chloroformnarcose anzusprechen. Jedoch fehlen darüber Erfahrungen.

2. Fall. 43 jährige Frau, hat $3 \mathrm{mal}$ geboren, $1 \mathrm{mal}$ abortirt. Eine Schwester in der Leipziger Anstalt an Manie mit Erfolg behandelt. Bereits im 17. Jahre geisteskrank, wurde $1 \%$ Jahre in der Prager Irrenanstalt behandelt. Zum 2. Mal im 32. Jahre psychisch erkrankt. Patientin kam jetzt in melancholischem Zustand in die Anstalt. Nach 6 Wochen stellten sich bei ihr klonische Krämpfe in der Gesichtsmuskulatur ein. Die Melancholie ging dann über in Manie.

Somatisch fand sich bei der Kranken ein gestielter Tumor der vorderen Uterusfläche aufsilzend. Die körperliche Abnahme liess auf einen maligneri T'umor schliessen. Die Krämpfe schienen in Zusammenhang mit dem Tumor zu stehen: bei jeder stärkeren Füllung des Unterleibes (Wassertrinken, Clysma) steigerten sich die Anfälle. Da die Kranke trotz künstlicher Ernährung sehr heruntergekommen war, wurde die Operation ausgeführt: Exstirpatio uteri cum adnexis. - Die Geschwulst sass breit auf, reichte tief in's Gewebe hinein. Unmittelbar nach der Operation war die Kranke ganz klar. Nach drei Tagen war sie jedoch bereits wieder verwirrt.

Antiseptischer Wundverlauf, trotzdem die Kranke den Verband mit Koth beschmierte. Die Erregung steigerte sich allmälig in den näcbsten 7 Wochen, blieb 7 Wochen auf der Höhe, ging dann langsam wieder zurück; ihr Zustand besserte sich so, dass sie in die Familie entlassen werden konnte.

Auf Grund dieser beiden Fälle legt Verfasser der Castration zum Zwecke der Heilung bei Neurosen resp. Psychosen grosses Gewicht bei.

Specielle Indicationen für die Operation lassen sich nicht aufstellen. Kann man aber nachweisen, dass eine Störung von den Genitalien aus reflectorisch auf die Gehirnthätigkeit wirkt, wäre solche Operation zu versuchen.

\section{Discussion.}

Mendel theilt einen Fall mit von bysterischer Psychose mässigen Grades. Da es sich um einen Ovarialtumor handelte, wurde die Ovariotomie gemacht. Verlauf ungünstig: die Kranke blieb daciernd geisteskrank. Mit der Schlussfolgerung des Verfassers stimmt er nicht ganz überein wegen der Schwierigkeit der Beurtheilung. Er erinnert an den Fall von Scheincastration, vorgetragen von Israel in der Bcrliner medicinischen Gesellschaft. Solche Fälle mahnen zur Vorsicht.

Flechsig hebt hervor, dass beide erwähnten Fälle sich unter seiner Statistik befinden. Bei dem Israel'schen Fallo ist es noch die Frage, in welchem Zustande sich die Genitalien befunden haben.

Hitzig berichtet über einen Fall von Castration bei Hysterie, welcher ungünstig verlaufen ist. 
Flechsig ist sich der Sohwierigkeiten in der Beurtheilung des jedesmaligen Falles bewusst; zur Operation müsste immer eine genügende Indication vorliegen.

No. 6. Richter-Dalldorf. Ueber die optischen Leitungsbabnen des menschlichen Gehirns.

Verfasser untersuchte post mortem drei Fälle von Atrophia nervi optici lateris unius und 6 Fälle von Atrophia nervi optici lateris utriusque.

1. Fall. Paralysis progressiva. Atrophie beider Optici. Bindegewebswucherung, vom Boden des 3. Ventrikels ausgehend, durch welches die Optici zur Atrophie gebracht wurden.

2. Fall. Allmälige Phthisis bulbi links. Erweichungsherd im rechten Hinterhauptslappen.

3. Fall. R. Atrophie des Opticus. Ein Psammom am rechten Opticus. Eine Erweichung im rechten Hirterhauptslappen.

In den anderen Fällen kein makroskopischer Befund. Mikroskopische Untersuchung der Fälle ergab: Schwund der Ganglienzellen im vorderen Vierbügel, im Seh- und Kniehügel beider Seiten.

Resultate: 1. Eine einseitige oder doppelseitige Sehnervenatrophie, welche das Gehirn eines Erwachsenen betraf, braucht sich in für das blosse Auge sichtbarer Weise über den gekreuzten Sehstreifen selbst nach 10 Jahren noch nicht zur Geltung zu bringen.

2. Innerbalb dieser Zeit wirkt die Sehnervenatrophie auf die gangliösen Gebilde der Kniehöcker, der oberen und unteren Vierhügel und der Sehhügel in gleicher Weise, und zwar wirkt einseitige Sehnervenatrophie auf die genannten Gebilde beider Seiten. Bei doppelseitiger Sehnervenatrophie cumulirt sich die Wirkung.

3. Zerfall des Occipitallappens bringt sich bezüglich der genannten vor ihm gelegenen Gebilde selbst nach Jahren noch nicht in für das blosse Aage sichtbarer Weise zur Geltung.

4. Ob Zerfall eines Oecipitallappens auf die Ganglienzellen der genannten vor ihm gelegenen Gebilde in gleicher Weise wirkt, wie Sehnervenatrophie, ist nicht erwiesen.

Eine Discussion fand nicht statt.

No. 7. Mendel-Berlin. Ueber die Ganglienzellen bei der propressiven Paralyse der Irren. (Vortrag ist veröffentlicht im Neurologischen Centralblatt 1884 No. 21).

Vortragender macht aufmerksam auf die Verschiedenheit des Aussehens der Ganglienzellen im normalen Gehirn. Veränderungen bei der Paralyse sind folgende:

1. Die pericellulären Lymphräume sind erweitert, zum Theil angefüllt mit einer gelblichen Flüssigkeit, welche durch Carmin gar nicht odor nur unerheblich gefärbt wird.

2. Fettig pigmentöse Degeneration in dem Protoplasma der Ganglienzellen.

3. Sklerose und Atrophie der Zellen. Der Inhalt wird streifig. DieZelle Arehiv f. Psyehiatrio. XVI. 2. Heft. 
glänzend. Kern verändert sich. Diese Form ist, wie Mendel nachgewiesen, experimentell herzustellen.

4. Veränderung des Kernes, sein Inhalt hat sich in derselben Weise, wie das Protoplasma verändert. Die Gestalt der Kerne ist dreieckig; sie liegen nicht, wie gewöhnlich, in der Mitte, sondern mehr am Rande. Zuweilen findet sich eine Vergrösserung des Kernes, so erheblich, dass derselbe die ganze Zelle ausfüllt.

In den Zellen der grossen Ganglien waren keine Veränderungen zu constatiren. wohl aber im Hypoglossuskern.

Obige Veränderungen sind nur an solchen Fällen von Paralyse beobachtet, die nach längerem Bestehen und nach Entwickelung hochgradiger De mentia und starken Lähmungserscheinungen den Exitus letalis nahmen.

Discussion.

Binswanger sieht für seine Untersuchungen als einen Vortheil an, dass or nur eine Gruppe von Ganglienzellen, nämlich die Betz'schen Rieserpyramidenzellen im Paracentrallappen ausgewählt hat. In einigen Punkten stimmt er mit den Mendel'schen Befunden überein: die pericellulären Lymphräume hat er nie angefüllt gesehen; deren Vorhandensein bezweifeli er nicht. Wiehtig sind für ihn die Veränderungen des Kernkörperchens (cfr. vorl. Mittheilung in den Verb. der Jenaischen Ges. für Med. u. Naturw. 11. Jan. und 13. Juni 1884). Die Kernveränderungen sind denen Mendel's analog. Zu beachten ist ferner, dass nur gewisse Gruppen von Ganglienzellen afficirt sind. Weiter schiebt er den Veränderungen des Pigments in den Ganglienzellen Bedeutung zu: Ursprünglich ist es dem Kern entgegegengesetzt, umlagert dann diesen und bedeckt ihn schliesslich. Bei normalen Präparaten kann man diese Verhältnisse deutlich erkennen; bei pathologischen ist es sehr schwierig zu unterscheiden, da Kern und Kernkörperchen häufig nicht deutlich zu erkennen sind.

v. Gudden hebt die Schwierigkeit der Untersuchungen hervor. Er erklärt auf Grund der Untersuchung eines Collegen, dass jedes in Müller'scher Flüssigkeit gehärtete Gehiru unbrauchbar sei, es müsse in Alkohol gehärtet sein. Je mehr vom Hirn uniersucht würde, desto besser die Resultate. Vergleichung mit normalen Präparaten und gleichmässige Behandlung wären vor Allem erforderlich. Nach ihm handelt es sich im wesentlichen um eine Atrophie der Zellen. Die Zelle will kleiner, klumpig, undurchsichtig, ihre Fortsälze werden undeutlich. Das Kernkörperchen verliert seinen Glanz.

Mendel weist den Vorwurf gegen die Müller'sche Lösung zurück, denn die Beschreibung der Verändeuungen des Vorredners an Alkololpräparaten stimmen mit den seinigen überein. Seine Behandlung war eine durchaus gleichmässige. In Bezug auf die Anfüllung der pericellulären Lymphräume weist Verf. auf die Seltenheit des Vorkommens hin: unter 3000 Präparaten hat er 11-12, welche dieses zeigen.

Binswanger erwähnt, dass Flemming seine Präparate zum Zwecke der Kernuntersuchung ausschliesslich in Chrom härtet.

Schluss der Sitzung.

Siemerling-Berlin. 